\title{
Immunohistochemical study on lipid catabolism in adult Onchocerca volvulus
}

\author{
Seidu Mahmood Abdulai ${ }^{1, ~ *, ~ A d a m s ~ A b d u l ~ R a s h i d ", ~ G y a s i ~ K w a m e ~ R i c h a r d ², ~ T e t t e y ~ Y a o ², ~}$ \\ Nkansah Obenewaa Dinah ${ }^{1}$, Adunyame Lois ${ }^{1}$, Wiredu Kwame Edwin ${ }^{1}$ \\ ${ }^{1}$ Department of Medical laboratory science, School of Allied Health Sciences, University of Ghana, Accra, Ghana \\ ${ }^{2}$ Department of Pathology, University of Ghana Medical School, Accra, Ghana
}

\section{Email address:}

amabdulai@chs.edu.gh (Seidu M. A.),raadams@chs.edu.gh (Adams A. R.),rkg539us@yahoo.com (Gyasi K. R.), tetteyyao@gmail.com (Tettey Y.), donkansah@ug.edu.gh (Nkansah O. D.), ladunyame@ug.edu.gh (Adunyame L.), ekwiredu@chs.edu.gh (Wiredu E. K.)

\section{To cite this article:}

Seidu Mahmood Abdulai, Adams Abdul Rashid, Gyasi Kwame Richard, Tettey Yao, Nkansah Obenewaa Dinah, Adunyame Lois, Wiredu Kwame Edwin. Immunohistochemical Study on lipid Catabolism in Adult Onchocerca Volvulus. American Journal of Biomedical and Life Sciences. Vol. 2, No. 4, 2014, pp. 65-69. doi: 10.11648/j.ajbls.20140204.11

\begin{abstract}
Onchocerca volvulus is a parasite responsible for the disease, Onchocerciasis otherwise known as river blindness. Current treatment and control strategies are not entirely successful. This means there is a need for further studies to better understand the biology of this worm. Studies on metabolic pathways in the worm can provide valuable information to deepen our understanding of the mechanism of survival of the worm. Lipid catabolism for the provision of energy in this worm still remains to be elucidated. Knowledge of lipids composition and synthesis in this parasite has been mostly drawn from studies on filarial species other than $O$. volvulus itself. This is owed to the limited availability of parasite material due largely to problems with parasite cultivation and ethical demands on obtaining materials since it has a strict preference for the human host. To explore lipid catabolism in the worm itself, we performed immunohistochemical localisation of three major enzymes (A/B hydrolase, SDH and ME1) involved in lipid metabolism on paraffin processed archival $O$. volvulus nodules. We observed that up to $58.6 \%$ of worms in the paraffin processed nodules had detectable A/B/HD4, 51.6\% had SDH and $63.3 \%$ had ME 1, most of which were stored in the muscles of the adult worm. These observations suggest that the adult $O$. volvulus can operate the B-oxidative pathway, The TCA cycle, and undergo anaplerotic transformation of malic acid to pyruvate to maximise energy production from lipid metabolism. This also suggests that $O$. volvulus has the capacity to catabolise lipids for energy even though it appears to be dependent on carbohydrate metabolism.
\end{abstract}

Keywords: Antibodies, Antigens, Onchocerciasis, Nodules, Enzymes

\section{Introduction}

Human Onchocerciasis, commonly known as river blindness, is a complex disease caused by the filarial worm, Onchocerca volvulus. It is a major cause of skin pathology and eye lesions including blindness, and has its highest prevalence in tropical Africa $[1,2,3,4,5]$.

Onchocerca volvulus is detected in two forms in humans: the larval stage (microfilaria), responsible for the blindness and the adult male or female stages (macrofilariae). Whereas the larvae (measuring between 220 to $360 \mathrm{um}$ ) move freely in the dermis of the skin, the much larger adults (measuring up to $5 \mathrm{~cm}$ for males and about $80 \mathrm{~cm}$ for females) are encapsulated in host fibrous tissues in the form of nodules $[4,5,6]$.
The treatment and eradication of Onchocerciasis has not been a complete success. This suggest that the search for a safe macrofilaricide against $O$. volvulus would continue to be a priority as it has been for the last two decades $[7,8]$. The ideal treatment requires drugs which will kill the adult worms (macrofilaricides) or permanently eliminate all microfilariae (microfilaricides), including those in gravid adult female worms in the infected individual $[8,9]$.

Studies on metabolic pathways in the parasite can yield information regarding the mechanisms for multiplication and survival of parasite as well as the disease process.

Evidence for the functioning of different metabolic pathways in parasites has been adduced mainly by the demonstration of the enzymatic steps or the identification of 
the intermediates of the pathways $[10,11]$.

Lipid catabolism for the production of energy in filarial worms is a relatively untouched aspect of the biology of filarial worms. Investigations have been restricted mainly to analyses of lipid composition and studies on lipid synthesis [12]. The incorporation of mevalonate into free fatty acids in filarial worms however suggests the presence of a fatty acid chain expansion system within filarial worms [13]. It is known that the worms readily incorporate labelled lipid precursors into their neutral and phospholipid fractions in their lipid bio-synthesis $[14,15,16]$. The entire major classes of neutral lipids namely, triacylglycerols, diacylglycerols sterols, sterol esters, hydrocarbons and traces of free fatty acids have been verified in Dirofilaria immitis $[13,16,17,18]$ while triacylglycerols and sterols have been demonstrated in adult Setaria cervi $[19,20]$. The adult Brugia pahangi has been reported to have triacylglycerols, sterols, as well as free fatty acids and hydrocarbon fractions in their tissues [18]. All the major phospholipid classes have also been identified in adult filarial worms of $D$. immitis, $B$. pahangi, Litomosoides carinii and $S$. cervi $[13,19,21,22]$. Glycer-ol-3-phosphate has been shown to participate in the formation of all phosphate related lipids in adult $B$. pahangi and B. patei [23]. These findings along with previous studies, suggest that filarial worms can synthesize almost all phosphate related lipids such as phosphatidylcholine, phosphatidylethanolamine and phosphatidylinositol and also fabricate phosphatidylserine, phosphatidylglycerol and cardiolipin [23] and for that matter are capable of degrading them for energy when there is a need to obtain energy from other sources different from their traditional source (carbohydrates).

Due to the problems associated with $O$. volvulus cultivation and the non-availability of materials since the worm's preference for humans impose strict ethical demands on obtaining their materials; $O$. volvulus has therefore not been extensively studied resulting in inadequate direct information on its biology [24] which is needed to understand its mechanisms of survival, hence the reliance on information from studies on closely related filarial species. By this project we could contribute to the direct appraisal of biological targets within the $O$. volvulus itself. This could avoid the transfer of knowledge from one species of worm to another which might not be applicable.

We recently demonstrated that a large number of long-time inappropriately stored paraffin embedded Onchocerca nodules retain enough antigenicity for immunohistochemical demonstration of some enzyme epitopes [25]. Based on this observation we performed immunohistochemical techniques on archival formalin-fixed paraffin processed $O$. volvulus nodules to demonstrate the presence of some metabolic enzymes involved in the breakdown of lipids for the provision of energy in the worm. The enzymes are al-pha/beta hydrolase -4 (A/BHD4), succinate dehydrogenase (SDH) and malic enzyme -1(ME1). The presence of these enzyme may be an indication that $O$. volvulus has the capacity to utilize lipids for energy when the need arises.

\section{Materials and Methods}

\subsection{Test Materials}

Thirty paraffin embedded $O$. volvulus nodules were obtained from Onchocerciasis Chemotherapy Research Centre (OCRC) in the Volta Region of Ghana, cleaned and re-embedded using fresh paraffin wax (Leica histowax, mp $57-58^{\circ} \mathrm{C}$; Leica Microsystems $\mathrm{GmbH}$, Germany) in freshly labeled cassettes as described in our previous publication [25]. All nodules had previously been fixed in $10 \%$ phosphate buffered formalin, dehydrated through ascending grades of ethanol and cleared in xylene. Sections from the re-embedded blocks were stained by haematoxylin and eosin method and examined by light microscopy to confirm the presence of adult $O$. volvulus in the tissues (Fig. 1). Immunohistochemical staining for enzymes A/BHD, SDH and ME1 were then performed on sections from these tissue blocks.

\subsection{Reagents}

Tris-Buffered Saline (TBS) x10 concentrate (Sigma T5912), 3, 3' dimethylaminobenzidine Tetrahydrochloride (DAB) (Sigma D 3939) consists of liquid buffer A (Sigma D6190) and liquid chromogen B (Sigma 6065), 30\% hydrogen peroxide (Sigma 95321) and Streptavidin-horseradish peroxidase polymer, Ultrasensitive (Sigma S2438)

\subsection{Buffers and Solutions}

Citrate buffer ( $\mathrm{pH}$ 6.0) (Sigma C2488), TBS ( $\mathrm{pH}$ 7.4) were obtained. Working solution of TBS contained: one part TBS x10 concentrate (Sigma T5912) and nine parts distilled water. Peroxidase-block solution $(0.03 \% \mathrm{H} 2 \mathrm{O} 2$ in $95 \%$ ethanol): 11 contains $3 \mathrm{ml}$ of $30 \% \mathrm{H} 2 \mathrm{O} 2$ and $997 \mathrm{ml}$ of $95 \%$ ethanol.

\subsection{Primary Antibodies}

Anti-Alpha/beta-hydrolase 4(Sigma HPA 000600)

Anti-Succinate dehydrogenase (Sigma HPA 002868).

Anti-Malic Enzyme (Sigma HPA 006493)

All reagents, buffers and antibodies were obtained from Sigma-Aldrich Chemie GmbH, (Taufkirchen, Germany).

\subsection{Staining Procedure}

The three sets of nodule sections labeled for A/BHD, SDH and ME1 and one negative control each were brought down to water and their antigens retrieved in citrate buffer $(\mathrm{pH}$ 6.0) using an ordinary floatation water bath at $65^{\circ} \mathrm{C}$ for 10 minutes[26]. Endogenous peroxidase was blocked for 5 minutes in a freshly prepared peroxidase block solution. They were placed on a humidified staining rack which has been described in our previous publication [26] and reagents applied in the following sequence: TBS ( 5 minutes), diluted primary antibodies (30 minutes), streptavidin-peroxidase polymer (30 minutes) and freshly prepared working DAB 
solution (10 minutes). The primary antibodies were applied to their appropriately labeled slides excluding the negative slides where TBS was maintained to avoid drying. The slides were rinsed and flooded with two changes of TBS for 5 minutes each after each application except after DAB where tap water was used to rinse the slides. The nuclei were stained with Mayer's haematoxylin for one minute and blued in tap water for five minutes. The sections were dehydrated in ethanol, cleared in xylene and mounted in DPX.

\subsection{Examination of Slides}

Sections were examined using an Olympus light microscope (Olympus CX31, model CX31RBSF). Negative staining showed no DAB reaction which was comparable with the negative controls while positive staining appeared as golden-brown to dark brown. The staining reactions were scored as follows: 0 - no reaction, $1+-$ mild reaction and $2+$ - strong reaction. The scoring was undertaken for the various cross-sectional anatomic sites of the $O$. volvulus sections (figure 1). The examination was carried out together by a biomedical scientist (MAS) and a pathologist (EKW) and both agreed on each score before it was recorded. Micrographs were captured using an Olympus digital camera (model DP20-50) mounted on an Olympus microscope (model BX51TF) and composited using a Microsoft Office power point Program and converted to JPEG images (figure 2).

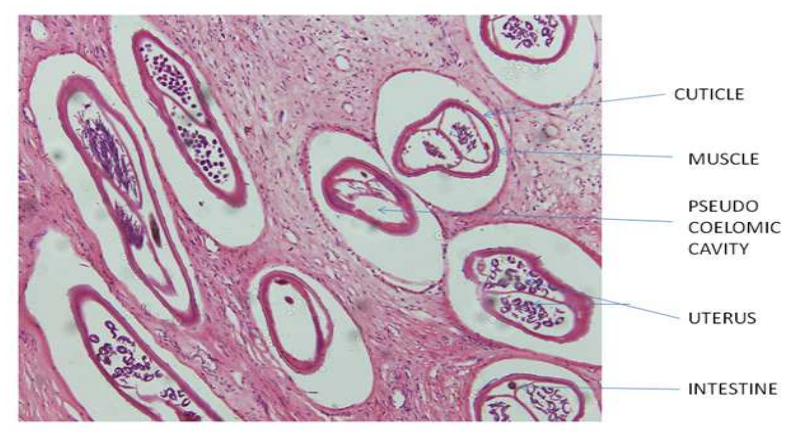

Figure 1. A photomicrograph showing the micro-anatomy of a cross-sectional view of a female Onchocerca volvulus in a paraffin section. $H \&$ E stain (x200 magnification)

\section{Results}

In all, 30 worms were scored in 30 nodules for Alpha/beta hydrolase $-4,33$ worms in 30 nodules for Succinate dehydrogenase and 31 worms in 30 nodules for malic enzyme 1. A male worm found in nodule number KP1 for SDH (KP1b) was not found in the corresponding slides stained with A/BHD4 and ME1. Similarly, a female worm found in nodule number KP16 for SDH was declared non-specific by both examiners and therefore not scored. Same as a female worm in nodule number KP19 for A/BHD4 who's staining was rejected as non-specific. Two male worms were found in slides stained for SDH (KP1 and
KP9) and one each for A/BHD4 and ME1 in KP9. No enzyme was detected in any of them. Generally, the enzymes were found mainly in the muscles of most of the worms and to a lesser extent in the walls of the genital tract and some microfilaria in the genital tracts. No enzyme deposits were found in the intestines.
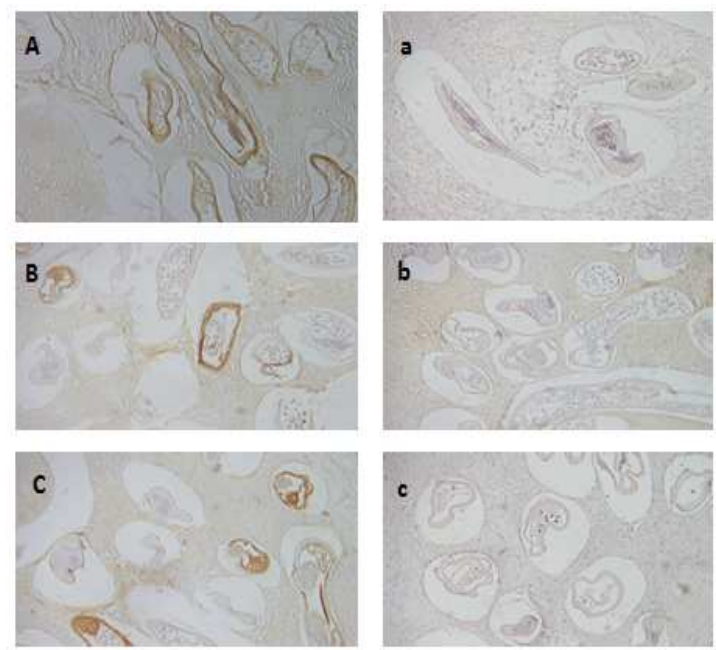

Figure 2. A photomicrograph of IHC stained of sections of $O$. volvulus showing A/BHD4 positive and negative A/a, SDH positive and negative $B / b, M E 1$ positive and negative $C / c$.

Table 1. Summary of results on A/BHD4, SDH and ME1

\begin{tabular}{lccc}
\hline ITEM & A/BHD4 & SDH & ME1 \\
\hline Total Number of worms & 30 & 33 & 31 \\
Number of male worms & 1 & 2 & 1 \\
Number of female worms scored & 29 & 31 & 30 \\
Enzyme positive female worms & 17 & 16 & 19 \\
Enzyme positive male worms & 0 & 0 & 0 \\
\% of female worm with enzyme & 58.6 & 51.6 & 63.3 \\
\hline
\end{tabular}

Table 2. Tests of proportional significance of A/BHD4, SDH and ME1 in O. volvulus (30\%, 35\% and 40\%) at 95\% and $99 \%$ levels of significance

\begin{tabular}{ccccccc}
\hline & NFW & Positive & \% & $\mathbf{0 . 3 0}$ & $\mathbf{0 . 3 5}$ & $\mathbf{0 . 4 0}$ \\
\hline A/BHD4 & 29 & 17 & 58.6 & $0.001 * *$ & $0.003 * *$ & $0.018^{*}$ \\
SDH & 31 & 16 & 51.6 & $0.005^{* *}$ & $0.031^{*}$ & 0.106 \\
ME1 & 30 & 19 & 63.3 & $0.001 * *$ & $0.007 * *$ & $0.005 * *$ \\
\hline
\end{tabular}

*Significant at $5 \% ; *$ Significant at $1 \%$

\subsection{Analysis of Results}

In our previous studies on archival nodules we showed that antigens in significant numbers of worms found in stored paraffin-embedded nodules maintain their Immunoreactivity even after poor storage for long periods [25]. However, since not all worms were found to have maintained the antigenicity of their epitopes, it was prudent to perform this study on several different nodules instead of relying on a single nodule. A test of proportions was done to indicate at what hypothesized proportions of the worms, the various enzymes could be found using (30\%, 35\% and 40\%) at $95 \%$ and $99 \%$ confidence level. The assumption made was that at least we expect to find a minimum of $30 \%$ of the worms or more in a sample with any of the enzymes. The 
test shows that we are $99 \%$ confident that a significant number of worms $(\geq 30 \%)$ will be found with all three enzymes ( $p$-values $=0.001,0.005,0.001)$. It can also be reported that at $99 \%$ confidence level a significant proportion $(\geq 35 \%)$ will be found with two of the enzymes (A/BHD and ME) with (p-values $=0.003$ and 0.007). At the same level of significance $(\geq 40 \%)$ of the worms will be found with only one enzyme (ME) with ( $p$-value=0.005). At a hypothesized proportion of $30 \%$ and $35 \%$ all three enzymes will be found in the worms at $95 \%$ confidence level (p-values $=0.001,0.005,0.001,0.003,0.031,0.007)$. The presence of one enzyme (SDH) in a group of worms was not significant at a hypothesized proportion of $40 \%$ at both confidence levels $(p$-value $=0.106)$

\section{Discussion}

The study was to demonstrate the presence and anatomical location of A/BHD (lipase), SDH and ME in adult $O$. volvulus embedded in paraffin wax for several years. It was clear that a significant number of worms maintained the antigenicity of all the enzymes. Alpha/beta hydrolase isoenzyme 4 was detected in $58.6 \%$ of the total number of female worms found in the nodules. This enzyme is responsible for breaking down fats (lipids) into fatty acids and glycerol. Fats (lipids) are one of the three major macronutrients needed for proper nutrition and lipase is an essential enzyme in the process. Although lipids are second to carbohydrates in terms of producing energy for living organisms, they by no means are a major form of energy storage in all organisms. The action of lipase breaks down lipids into fatty acids and glycerol allowing the fatty acids to participate in a beta oxidation process that eventually generates energy for the organism. The main product of this pathway is the generation of several copies of acetyl-CoA which ultimately enters the tri-carboxylic acid (TCA) cycle as a convenient conversion point with the carbohydrate catabolic processes.

Succinate dehydrogenase (SDH) was detected in $51.6 \%$ of all the female worms. In human cells SDH enzymes plays a key role in the process of oxidizing nutrients to release energy when oxygen is present. One of the important functions of succinate dehydrogenase is its activity on succinate which converts it to fumarate, a process that results in the production of energy for organisms that have the enzyme. This process also facilitates one of the vital steps in the TCA cycle by regenerating fumarate a precursor to oxaloacetate which combines with acetyl-CoA to initiate another round of TCA. The presence of SDH in O. volvulus comes with several metabolic options under aerobic conditions. Under this condition, pyruvate and essentially acetyl-CoA are substrates to the TCA whose activity can be operative or terminated by the presence or absence of SDH respectively.

Malic enzyme (ME) was detected in $63.3 \%$ of all the female worms. This enzyme catalyzes the oxidative decarboxylation of malate to pyruvate. The presence of this enzyme in $O$. volvulus creates an opportunity for the worm to avoid a possible inactivation of malate dehydrogenase which is essential in the regeneration of oxaloacetate after the activity of succinate dehydrogenase.

Onchocerca volvulus is unable to survive in any other vertebrate host except humans and attempts to transplant Onchocerca nodules into chimpanzees for experimental purposes were not successful [27]. The general understanding from various studies is that most filarial worms have active trans-cuticular absorption mechanisms which allow them to absorb nutrients and other useful life supporting molecules from their hosts [28]. In O. volvulus this mechanism has been found to be a continuous process as a result of its reluctance to store more than $2 \%$ of its wet body weight as carbohydrates which it uses consistently [12]. In terms of survival it appears that beta oxidation is the likely path for degradation of fatty acids in the worm. This eventually results in the production of acetyl-CoAs and an inevitable entry into the TCA cycle. By the presence of alpha/beta hydrolase (lipase) in $O$. volvulus, it can be said that it has the capacity to break down lipids to fatty acids and fatty acid to energy.

We were unable to detect any of the enzymes in the male worms found in the nodules, although both appeared histologically viable. We cannot offer a definite explanation for this finding but we believe that the male worms may have been affected by anoxia, which might have led to the release of enzymes during delays in their preservation since they were found deep in the central parts of the nodules. The presence of these three enzymes in a significant number of the worms show that adult $O$. volvulus are capable of using lipids to generate energy when carbohydrates catabolic processes are inoperative due to lack of carbohydrate or inhibition of the process. This could allow the worms to diverge from chemotherapeutic inhibition of one pathway to a less affected pathway during the period of inhibition of their more preferred carbohydrate pathways.

\section{Conclusion}

This study suggests that the adult $O$. volvulus can operate the beta oxidative pathway, the TCA and perform an anaplerotic diversion of malic acid to pyruvate. Furthermore $O$. volvulus has the capacity to catabolise lipids for energy provision even though it appears to be dependent on carbohydrate metabolism.

\section{Acknowledgements}

The reagents were purchased by the University Of Ghana School Of Allied Health Sciences through their local research funds; we thank the Dean of the School and the head of department of Medical Laboratory Sciences for the support. We are also grateful to the late Dr. K. Awadzi, the former director of Onchocerciasis Chemotherapy Research Centre at Hohoe hospital in Ghana and his staff for the Onchocerca volvulus nodules. Finally, we would like to 
thank Mr. D. Nana Adjei of the University of Ghana School Of Allied Health Sciences for helping us in the statistical analysis.

\section{References}

[1] Murdoch, M. E., Hay, R. J., MacKenzie, C. D., Williams, J. F., Ghalib, H. W., Cousens, S., Abiose, A., \& Jones, B. R. A clinical classification and grading system of the cutaneous changes in onchocerciasis. British Journal of Dermatology.1993; 129(3): 260-269.

[2] Burnham, G. Onchocerciasis. Lancet.1998; 351(9112): $1341-1346$

[3] Hoerauf, A., Bütmer, D. W., Adjei, O., \& Pearlman, E. (2003). Science, medicine, and the future: Onchocerciasis. British Medical Journal.2003; 326(7382): 207-210

[4] Brattig, N. W. Pathogenesis and host responses in human onchocerciasis: Impact of Onchocerca filariae and Wolbachia endobacteria. Microbes and Infection.2004; 6(1): 113-128

[5] Bradley, J. E. Onchocerciasis. In F. E. G. Cox, J. P. Kreier \& D. Wakelin (Eds.), Topley \& Wilson's Microbiology \& Microbial Infections: Parasitology. Washington, D.C.: ASM Press.2005;10th ed., pp. 781-801

[6] Enk, C. D. Onchocerciasis-river blindness. Clinics in Dermatology.2006; 24(3): 176-180

[7] WHO, Twenty years of Onchocerciasis control in West Africa: review of the work of Onchocerciasis control programme in West Africa from 1974 to 1994.1995

[8] Alley, W. S., Oortemarssen, G. J., Boakye, A. B., Nico, J. D. N., Plaisier, P. A. Femme, J. H. F., Lazdins, J., Borsboom, G. J. J. M., and Habbema, J. D. F. Macrofilaricides and onchocerciasis, mathematical modelling of the prospect for elimination. BioMed Central Ltd.2001

[9] Forgione Michael A. Jr. Introduction to clinical differentials of Onchocerciasis. Excerpt from Medicine.com, Inc (abstracts) 2002.

[10] Aloysius G.M. Tielens, Koen W.A. van Grinsven, Katrin Henze, Jaap J. van Hellemond, William Martinca. Acetate formation in the energy metabolism of parasitic helminths and protists. International Journal for Parasitology. 2010; 40: $387-397$

[11] Arumugam S, Hoerauf A, Pfarr K. M. Localization of a filarial phosphate permease that is up-regulated in response to depletion of essential Wolbachia endobacteria. Exp Parasitol. $2014 ; 138: 30-9$

[12] Barrett, J. Biochemistry of filarial worms. Helmintholgical Abstracts. Ser. A, Animal and human helminthology.1983; 52: $1-18$

[13] Comley J.C.W. and Jaffe J.J. Isoprenoid biosynthesis in adult Brugia pahangi and Dirofilaria immitis. Journal of Parasitology.1981; 67: 609-616

[14] Jaffe, J. J. and Doremus, H. M. Metabolic patterns of Dirofilaria immitis microfilariae in vitro. Journal of Parasitology.1970; 56: 254.260.
[15] Hutchison, W. F. and Turner, A.C. Glycerol metabolism in the adult dog heartworm, Dirofilaria immitis. Comparative Biochemistry and Physiology, B, Comparative Biochemistry.1979; 64; 399-401.

[16] Turner A. C. and Hutchison W. F. Lipid synthesis in the adult dog heartworm, Dirofilaria immitis. Comparative biochemistry and Physiology B, Comparative Biochemistry.1979; 64: 403-405

[17] Hutchison, W. F., Turner, A. C., Grayson, D. P. and White H.B. Lipid analysis of the adult dog heartworm, Dirofilaria immitis. Comparative Biochemistry and Physiology, B, Comparative Biochemistry.1976; 53: 495.497

[18] Comley J.C.W., Jaffe J.J., Chrin L.R. and Smith R.B. Synthesis of ubiquinone 9 by adult Brugia pahangi and Dirofilaria immitis: evidence against its involvement in the oxidation of 5-methyltetrahydrofolate. Molecular and Biochemical Parasitology.1981; 2: 271.283

[19] Ansari, A. A., Yusufi, A. N. and Siddiqi, M. Lipid composition of the filarial parasite Setaria cervi. Journal of Parasitology.1973; 59:939.940.

[20] Rathaur, S., Anwar N. and Ghatak, S. Biochemical composition of microfilarial and adult stages of Setaria cervi. Zeitschrift für Parasitenkunde.1980; 62: 85.93

[21] Hack, M. G., Gussin, A. E. and Lowe M.E. Comparative lipid biochemistry-I. Phosphatides of invertebrates (Porifera to Chordata).Comparative Biochemistry and Physiology.1962; 5: $217-221$

[22] Subrahmanyam D. Glyceral ether phospholipids and plasmalogens of filarial parasite Litomosoides carinii. Canadian Journal of Biochemistry.1967; 45: 1195-1197.

[23] Srivastava, A. K., Walter, R. D. and Jaffe J.J. Synthetic pathways of glycerophospholipids in adult Brugia pahangi and Brugia patei. International Journal for Parasitology.1987; $17 ; 1321.1328$.

[24] Lizotte-Waniewski, M., Tawe, W., Guiliano, D. B., Lu, W., Liu, J., Williams, S. A and Lustigman, S. Identification of potential Vaccine and Drug Target Candidates by Expressed Sequence Tag Analysis and immunoscreening of Onchocerca volvulus larval cDNA libraries. Infection and immunity.2000; 68 (6): 3491-3501

[25] Seidu, M.A., Adams, A.R., Gyasi, R.K., Tettey, Y. Nkansh, D.O., Wiredu. E.K. Immunoreactivity of some epitopes in longtime inappropriately stored paraffin embedded Tissues. Journal of Histotech. 2013;36(2): 59-64

[26] Seidu, M.A., Adams, A.R., Gyasi, R.K., Tettey, Y Wiredu, E.K. Short time incubation at low temperature for retrieval of some antigens from formalin-fixed and paraffin-embedded tissues. Journal of Histotech.2012; 35(2):74-79.

[27] Buttner, D.W., Albiez, E.J., von Essen, J. and Erichsen, J. Histological examination of adult Onchocerca volvulus and comparison with the collagenase technique. Trop. Med. Parasit.1988; 39: 390-417

[28] Kohler, P. The pathways of energy Generation in Filarial Parasites. Parasitology Today.1991; 7(1): 21-24. 